\title{
Pre- and post-bronchodilator airway obstruction are associated with similar clinical characteristics but different prognosis - report from a population-based study
}

\author{
This article was published in the following Dove Press journal: \\ International Journal of COPD \\ 24 April 2017 \\ Number of times this article has been viewed
}

\author{
Sami Sawalha' \\ Linnea Hedman ${ }^{2}$ \\ Eva Rönmark ${ }^{2}$ \\ Bo Lundbäck ${ }^{3}$ \\ Anne Lindberg' \\ 'Department of Public Health \\ and Clinical Medicine, Division of \\ Medicine, ${ }^{2}$ Department of Public \\ Health and Clinical Medicine, The \\ OLIN Unit, Division of Occupational \\ and Environmental Medicine, \\ Umeå University, Umeå, ${ }^{3}$ Krefting \\ Research Center, Institution of \\ Medicine, University of Gothenburg, \\ Gothenburg, Sweden
}

\begin{abstract}
Introduction: According to guidelines, the diagnosis of COPD should be confirmed by postbronchodilator (post-BD) airway obstruction on spirometry; however, in clinical practice, this is not always performed. The aim of this population-based study was to compare clinical characteristics and prognosis, assessed as mortality, between subjects with airway obstruction divided into pre- but not post-BD obstruction, post-BD airway obstruction (COPD), and subjects without airway obstruction.
\end{abstract}

Materials and methods: In 2002-2004, four adult population-based cohorts were reexamined with spirometry and structured interview. Subjects with airway obstruction, with a ratio of forced expiratory volume in $1 \mathrm{~s}$ to (forced) vital capacity $<0.70$ ( $\mathrm{n}=993)$, were identified together with sex- and age-matched referents $(n=993)$. These subjects were further divided into subjects with pre- but not post-BD airway obstruction (pre- not post-BD obstruction) and subjects with post-BD airway obstruction (COPD). Mortality data were collected until December 31, 2014.

Results: Out of 993 subjects with airway obstruction, 736 (74\%) had COPD and 257 (26\%) pre- not post-BD obstruction. Any respiratory symptoms, allergic rhinitis, asthma, exacerbations, and comorbidities were equally common among subjects with COPD and pre- not post-BD obstruction, but less common among nonobstructive subjects. Mortality was highest among subjects with COPD and higher in men than in women. In both sexes, COPD, but not pre- not post-BD obstruction, was associated with an increased risk for death compared to those without airway obstruction. When COPD was divided into Global Initiative for Chronic Obstructive Lung Disease (GOLD) stages, GOLD 2 and 3-4 had an increased risk for death when compared to the nonobstructive group, also when adjusted for common confounders and comorbidities such as heart disease, diabetes, and anxiety/depression.

Conclusion: Even though subjects with COPD and pre- not post-BD obstruction had fairly similar presentation of clinical characteristics, only those with COPD, specifically GOLD stage $\geq 2$, had increased risk for death when compared with nonobstructive subjects.

Keywords: epidemiology, spirometry, chronic airflow obstructions, mortality

\section{Introduction}

Of the two major obstructive airway diseases, neither COPD nor asthma is considered as single diseases, but heterogeneous and complex syndromes. ${ }^{1}$ According to the Global Initiative for Chronic Obstructive Lung Disease (GOLD) document, respiratory symptoms, exacerbations, and obstructive lung function impairment are evaluated when assessing severity of COPD. ${ }^{2}$ GOLD recommends a post-bronchodilator (post-BD)
Correspondence: Sami Sawalha Department of Respiratory Medicine, Sunderby Hospital, Sjukhusvägen 10 SE 95442, Södra Sunderbyn, Sweden Tel +46762003 582

Emailsamisawalha@yahoo.com 
fixed ratio, forced expiratory volume in $1 \mathrm{~s}\left(\mathrm{FEV}_{1}\right) /$ forced vital capacity $(\mathrm{FVC})<0.70$, to define airway obstruction, whereas the lower limit of normal (LLN) nowadays is recommended in epidemiological studies. ${ }^{3}$ Still, spirometric criteria in most current guidelines for treatment of COPD are based on the fixed ratio to define COPD and on $\mathrm{FEV}_{1}$ percent of predicted to define severity.

However, in clinical practice, spirometry is an underutilized diagnostic method contributing to the well-known underdiagnosis of COPD. ${ }^{4-6}$ Moreover, post-BD spirometry is not always performed, ${ }^{7-9}$ and the use of merely pre-bronchodilator spirometry could misclassify nearly one-third of nonobstructive subjects as obstructive..$^{7-10}$ In addition to this diagnostic dilemma, there is lack of knowledge about the importance of pre-respectively post-bronchodilation airflow limitation in relation to the clinical picture and prognosis of obstructive airway diseases. ${ }^{11}$ Also, the presence of comorbid conditions is important when evaluating clinical characteristics in relation to prognosis. ${ }^{12-15}$ Comorbidities are common in COPD, and it has been estimated that $68 \%$ of subjects with COPD have cardiovascular disease, diabetes, or depression. ${ }^{16}$ Furthermore, though it has been indicated that women are more susceptible to the harmful effects of tobacco smoke exposure, ${ }^{15}$ there are few studies evaluating possible sex differences within this context.

The aim of this population-based study was to identify the extent of misclassification due to use of pre-BD spirometry in COPD diagnostics. Further, the study compared clinical characteristics and prognosis, assessed as mortality, of subjects with post-BD airway obstruction (COPD), prebut not post-BD airway obstruction, and subjects without airway obstruction.

\section{Materials and methods Study population}

The study design and recruitment of study population have previously been described in detail. ${ }^{17}$ Four adult populations-based cohorts within the epidemiological research program Obstructive Lung Disease in Northern Sweden (OLIN) were reexamined during 2002-2004; all subjects with $\left(\mathrm{FEV}_{1} / \mathrm{FVC}<0.70\right)$ were identified $(\mathrm{n}=993)$, together with age- and sex-matched controls without obstructive lung function impairment. Since 2005, the study population $(n=1,986)$ has been invited to annual examinations. Crosssectional data from baseline examinations in 2002-2004 were used in this paper together with mortality data collected from the Swedish national mortality register from the date of examination at recruitment until December 31,
2014. The study was approved by the Regional Ethics Committee at Umeå University. All participants provided informed consent.

\section{Structured interview}

A questionnaire including well-validated questions regarding respiratory symptoms and conditions was used. ${ }^{18-20}$ In addition, data on age, height, weight, smoking habits, and comorbid conditions were collected.

\section{Definitions}

Age group: $\leq 60$ and $>60$ years of age. Smoking habits were classified into the following groups: nonsmokers, ex-smokers (quit smoking since at least 1 year), current smokers, and ever smokers (current or ex-smoker). Smoking habits were missing in 22 subjects, and in these cases classified based on smoking habits at previous and past examinations. One pack-year: an equivalent to 20 cigarettes smoked every day for 1 year. Productive cough: cough and phlegm most days during at least 3 months during the last 12 months. Dyspnea was classified according to the modified Medical Research Council (mMRC) dyspnea scale (grades 1-4). ${ }^{21,22}$ Wheeze: have you at any time during the last 12 months had wheezing or whistling in your chest? Any respiratory symptoms: at least one of the symptoms - long-standing cough, productive cough, wheeze, or $\mathrm{mMRC}$ score $\geq 2$. Allergic rhinitis: selfreported hay fever or allergic rhinoconjunctivitis. Asthma: physician diagnosis of asthma before the age of 40. Any exacerbation: contacted health care due to respiratory complaints during the last 12 months. Frequent exacerbations: two or more such events during the last 12 months. Heart disease: angina pectoris, previous coronary artery bypass surgery, previous percutaneous coronary intervention, myocardial infarction, or heart failure. Diabetes mellitus: have or have had diabetes mellitus. Anxiety/depression: have or have had anxiety and/or depression.

\section{Spirometry and spirometric classification}

Lung function testing was performed using the dry volume spirometer, Mijnhardt Vicatest 5, in accordance with American Thoracic Society guidelines. ${ }^{23}$ Vital capacity (VC) was defined by using the best value of FVC and slow VC. All subjects with $\mathrm{FEV}_{1} / \mathrm{VC}<0.70$ or $\mathrm{FEV}_{1}<90 \%$ of predicted value performed post-BD spirometry after 4 doses of salbutamol $0.2 \mathrm{mg}$.

The following definitions were used. Non obstruction: pre-BDFEV ${ }_{1} / \mathrm{VC} \geq 0.70$. Airway obstruction: $\mathrm{FEV}_{1} / \mathrm{VC}<0.70$, further divided into pre- but not post-BD airway obstruction 
(pre- not post-BD obstruction) and post-BD airway obstruction (post-BD obstruction). Post-BD obstruction, $\mathrm{FEV}_{1} / \mathrm{VC}<0.70$, was based on the highest values of pre- or post-bronchodilation, that is, fulfilling the spirometric criteria for COPD and thus hereafter labeled COPD. The distribution of study population by spirometric definitions is shown in Figure 1. The COPD group was further classified by severity of airflow limitation based on $\mathrm{FEV}_{1}$ percent of predicted according to GOLD, into GOLD $1-4 .^{2}$ The Swedish OLIN reference values based on a nonsmoking respiratory healthy population were used. ${ }^{24}$

\section{Statistical analysis}

The Statistical Package for Social Sciences software version 20 (IBM, New Castle, NY, USA) was used for statistical analyses. A $P$-value $<0.05$ was considered statistically significant. The chi-square test was used for comparing proportions in bivariate analysis and trends and independent sample $t$-test for comparing means. Missing answers in individual questions were uncommon, $<0.5 \%$. Due to few subjects having severe and very severe COPD, GOLD 3 and 4 were grouped together. Mortality was based on at least 10 years observation time from examination date at recruitment in 2002-2004 until December 31, 2014. Survival was illustrated by Kaplan-Meier curves for the groups nonobstructive, prenot post-BD obstructive, and COPD, and also when COPD was divided into GOLD grades. Risk factors for death were analyzed by multivariate Cox regression and expressed as hazard ratio (HR) using 95\% confidence intervals (CIs) for statistical significance, covariates were spirometric groups

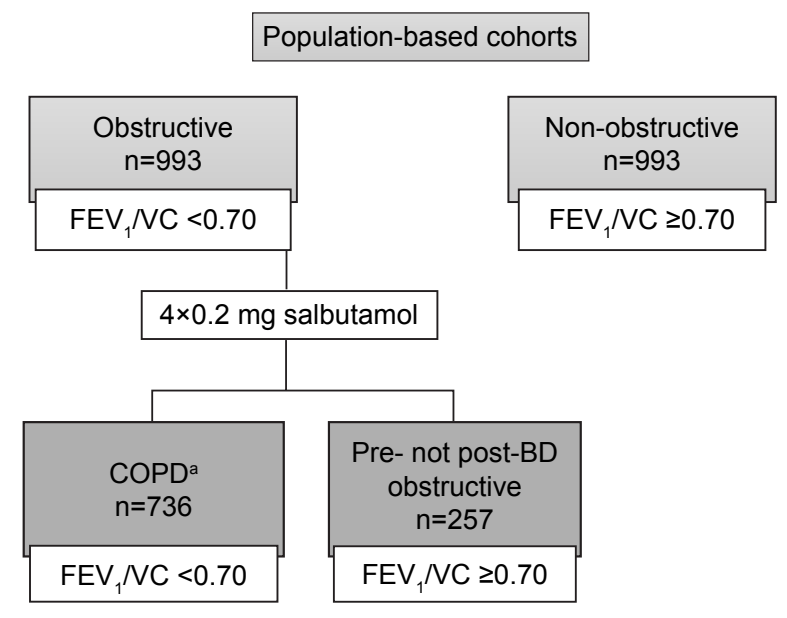

Figure I Study population by spirometric classification.

Note: aPost-BD obstructive; fullfilling the spirometric criteria for COPD according to GOLD.

Abbreviations: $\mathrm{BD}$, bronchodilator; $\mathrm{FEV}$, forced expiratory volume in I s; GOLD, Global Initiative for Chronic Obstructive Lung Disease; VC, vital capacity. (nonobstructive, pre- not post-BD obstructive, and COPD), age, sex, body mass index (BMI), and smoking habits. Analyses were also carried out in a model where COPD was divided into GOLD grades. Each of the variables heart disease, anxiety/depression, and diabetes mellitus was added to the model one by one in separate analyses, and finally also together. Sex-stratified analyses of risk factor for death were performed in corresponding models.

\section{Results}

\section{Baseline characteristics of the study population}

There were 993 subjects without airway obstruction, and 993 subjects with airway obstruction, whereof $26 \%(n=257)$ with pre- not post-BD obstruction and $74 \%(n=736)$ with post-bronchodilation airway obstruction (COPD) (Figure 1). Subjects with COPD were distributed by GOLD grades 1,2 , and 3-4 as follows: $37.0 \%(n=272), 53.5 \%(n=394)$, and $9.5 \%(n=70)$. Any respiratory symptoms, allergic rhinitis, physician diagnosis of asthma before the age of 40 , exacerbations, and comorbidities were equally common among subjects with COPD and pre- not post-BD obstruction, while productive cough and smoking were more common among subjects with COPD. Both among subjects with pre- not post-BD obstruction and COPD most respiratory conditions were more common than among nonobstructive subjects (Table 1).

Corresponding analyses stratified by sex are shown in Table 2. When comparing smoking habits and prevalence of respiratory conditions between COPD, pre- not post-BD obstructive, and nonobstructive patients, men and women had a similar pattern. Among women, there was no difference in prevalence of comorbid conditions between the groups, while among men, heart disease and anxiety/depression were more common among subjects with COPD compared with among nonobstructive, and anxiety/depression was also more common among pre- not post-BD obstruction than among nonobstructive subjects.

Men and women were compared within each of the groups; COPD, pre- not post-BD obstructive, and nonobstructive in Table 3. Any respiratory symptoms, exacerbations, and physician diagnosis of asthma before the age of 40 were equally common among men and women in all groups. Smoking habits differed by sex; men had a higher proportion of ever smokers in all groups. Allergic rhinitis was more common among women with COPD and pre- not post-BD obstruction and $\mathrm{mMRC} \geq 2$ was more common among women in all groups. Heart disease and diabetes were more common 
Table I Basic characteristics of the study population at recruitment, comparing COPD, pre- not post-BD obstructive, and nonobstructive subjects

\begin{tabular}{|c|c|c|c|c|c|c|}
\hline \multirow[t]{4}{*}{ Variables } & \multicolumn{3}{|c|}{ Airway obstruction $^{a}$} & \multicolumn{3}{|c|}{ Nonobstructive $^{\text {b }}$} \\
\hline & \multirow{3}{*}{$\begin{array}{l}\text { COPD } \\
n=736\end{array}$} & \multirow{3}{*}{$\begin{array}{l}\text { Pre- not post-BD } \\
\text { obstruction }^{c} \\
\mathrm{n}=257\end{array}$} & \multirow[t]{3}{*}{$P$-value ${ }^{d}$} & & \multirow[t]{3}{*}{$P$-value ${ }^{e}$} & \multirow[t]{3}{*}{$P$-value ${ }^{f}$} \\
\hline & & & & & & \\
\hline & & & & $\mathrm{n}=993$ & & \\
\hline Age mean $( \pm S D)$ & $65.4(11.0)$ & $63.3(12.3)$ & 0.012 & $64.5(11.3)$ & 0.088 & 0.117 \\
\hline BMI mean $( \pm S D)$ & $25.7(3.9)$ & $27.0(4.6)$ & $<0.001$ & $26.6(3.8)$ & $<0.001$ & 0.165 \\
\hline $\mathrm{FEV}_{1} \%$ pred $($ mean $\pm \mathrm{SD})$ & $73.3(17.4)$ & $84.5(14.1)$ & $<0.001$ & $92.8(13.0)$ & $<0.001$ & $<0.001$ \\
\hline Nonsmoker & $166(22.6)$ & $72(28.0)$ & & $475(47.8)$ & & \\
\hline Ex-smoker & $291(39.5)$ & I33 (5 I.8) & & $392(39.5)$ & & \\
\hline Current smoker & $279(37.9)$ & $52(20.2)$ & $<0.001$ & $126(12.7)$ & $<0.001$ & $<0.001$ \\
\hline Ever smoker & $570(77.4)$ & $185(72.0)$ & 0.077 & $518(52.2)$ & $<0.001$ & $<0.001$ \\
\hline Pack year $($ mean $\pm S D)$ & $16.6(16.7)$ & II.6 (I4.8) & $<0.001$ & $6.9(11.2)$ & $<\mathbf{0 . 0 0 1}$ & $<0.00 \mathrm{I}$ \\
\hline Productive cough & $338(45.9)$ & 87 (33.9) & 0.001 & $233(23.5)$ & $<0.001$ & 0.001 \\
\hline $\mathrm{mMRC} \geq 2$ & $209(28.4)$ & $54(21.0)$ & 0.021 & $94(9.5)$ & $<0.001$ & $<0.001$ \\
\hline Any respiratory symptoms & $55 \mid(75.0)$ & $180(70.0)$ & 0.123 & $432(43.5)$ & $<0.001$ & $<0.001$ \\
\hline Allergic rhinitis & $209(28.5)$ & $84(32.7)$ & 0.203 & $244(24.6)$ & 0.137 & 0.009 \\
\hline Physician diagnosis of asthma & $82(11.1)$ & $36(14.0)$ & 0.466 & $37(3.7)$ & $<0.001$ & $<0.001$ \\
\hline Exacerbation, any & $160(21.7)$ & 46 (17.9) & 0.191 & $89(9.0)$ & $<0.001$ & $<\mathbf{0 . 0 0 I}$ \\
\hline Exacerbations, frequent & $66(9.0)$ & $19(7.4)$ & 0.437 & $30(3.0)$ & $<0.001$ & 0.001 \\
\hline Heart disease & $139(18.9)$ & $46(17.9)$ & 0.726 & $160(16.1)$ & 0.132 & 0.492 \\
\hline Diabetes mellitus & $66(9.0)$ & $25(9.7)$ & 0.716 & $98(9.9)$ & 0.249 & 0.946 \\
\hline Anxiety/depression & $136(18.5)$ & $41(16.0)$ & 0.363 & $134(13.5)$ & 0.005 & 0.311 \\
\hline Mortality & $268(36.4)$ & 73 (28.4) & 0.020 & $262(26.4)$ & $<0.001$ & 0.515 \\
\hline
\end{tabular}

Notes: Data presented as n (\%) unless stated otherwise. Significant $P$-values in bold. ${ }^{a} F E V$, best of FVC and VC $<0.70$; ${ }^{\text {} F E V ~} /$ best of FVC and VC $\geq 0.70 ;{ }^{c}$ pre- not post-BD obstructive; 'comparing COPD and pre- not post-BD obstructive; ' ${ }^{\mathrm{d}}$ comparing COPD and nonobstructive; 'comparing pre- not post-BD obstructive and nonobstructive. Abbreviations: BD, bronchodilator; BMI, body mass index; FEV ${ }_{1}$, forced expiratory volume in I s; FVC, forced vital capacity; mMRC, modified Medical Research Council; pred, predicted; SD, standard deviation; VC, vital capacity.

among men, while anxiety/depression was more common among women in the groups COPD and nonobstructive.

\section{Mortality among COPD, pre- not post-BD obstructive, and nonobstructive}

In total, 603 subjects, 370 men and 233 women, had deceased during the follow-up time. Mortality was higher among subjects with COPD, 36.4\%, compared with pre- not post-BD obstructive, $28.4 \%$, but similar when comparing those with prenot post-BD obstruction and nonobstructive, 26.4\% (Table 1). A similar pattern was observed in the analyses stratified by sex (Table 2), while men had higher mortality than women among COPD and nonobstructive subjects (Table 3). Kaplan-Meier curves illustrate survival for the groups nonobstructive, pre- not post-BD obstructive, and COPD (Figure 2A) and also when COPD was divided into GOLD grades (Figure 2B).

\section{Risk for death; COPD and pre-BD obstructive compared with nonobstructive}

In unadjusted analyses, subjects with COPD but not pre- not post-BD obstructive had increased risk for death, HR 1.48 (95\% CI 1.24-1.75) when compared with nonobstructive subjects, likewise in men and women and those $>60$ years of age, while among subjects $\leq 60$ years of age at recruitment, those with pre- not post-BD obstruction had an increased risk for death (Table 4). When subjects with COPD were divided into GOLD grades, GOLD 2 and GOLD 3-4 had an increased risk for death with a similar pattern in men, women, and subjects $>60$ years of age, while among subjects $\leq 60$ years only those with GOLD $3-4$ had an increased risk for death (Table 4).

When analyzed in a Cox regression model adjusting for age, sex, smoking habits, and BMI classes, the increased risk for death, HR (95\% CI), associated with COPD remained, 1.24 (1.04-1.49), with a similar pattern among men, 1.27 (1.00-1.60), and women, 1.24 (0.92-1.13). In analyses stratified by age group, $\leq 60$ and $>60$ years, the increased risk for death among subjects with pre- not post-BD airway obstruction aged $\leq 60$ years disappeared.

The increased risk for death, HR $(95 \% \mathrm{CI})$, associated with COPD persisted also after including comorbidities (heart disease, diabetes mellitus, and anxiety/depression) in the model; $1.23(1.03-1.47)$ and the pattern was similar in men 1.23 (0.98-1.55) and women 1.24 (0.93-1.67). When dividing COPD by GOLD grades, GOLD 2 and GOLD 3-4 
Table 2 Basic characteristics among men and women at recruitment, comparing the groups COPD, pre- not post-BD obstructive, and nonobstructive

\begin{tabular}{|c|c|c|c|c|c|c|}
\hline \multirow[t]{4}{*}{ Variables } & \multicolumn{3}{|c|}{ Airway obstruction $^{a}$} & \multicolumn{3}{|c|}{ Nonobstructive $^{b}$} \\
\hline & \multirow{3}{*}{$\begin{array}{l}\text { COPD } \\
n=403\end{array}$} & \multirow{3}{*}{$\begin{array}{l}\text { Pre- not post-BD } \\
\text { obstructionc }^{c} \\
n=139\end{array}$} & \multirow[t]{3}{*}{$P$-value ${ }^{d}$} & & \multirow[t]{3}{*}{$P$-value ${ }^{e}$} & \multirow[t]{3}{*}{$P$-value } \\
\hline & & & & & & \\
\hline & & & & $n=542$ & & \\
\hline \multicolumn{7}{|l|}{ Men } \\
\hline Age $($ mean $\pm S D)$ & $65.5(10.7)$ & $61.6(12.8)$ & 0.001 & $64.1(11.3)$ & 0.057 & 0.034 \\
\hline $\mathrm{BMI}($ mean $\pm \mathrm{SD})$ & $25.9(3.5)$ & $27.3(4.4)$ & 0.001 & $26.6(3.4)$ & 0.005 & 0.56 \\
\hline $\mathrm{FEV}_{1} \%$ pred $($ mean $\pm \mathrm{SD})$ & $72.8(17.6)$ & $84.1(13.4)$ & $<0.001$ & $92.4(12.9)$ & $<0.001$ & $<0.001$ \\
\hline Nonsmoker & $68(16.9)$ & $29(20.9)$ & & $210(38.7)$ & & \\
\hline Ex-smoker & $189(46.9)$ & $83(59.7)$ & & $273(50.4)$ & & \\
\hline Current smoker & $146(36.2)$ & $27(19.4)$ & 0.001 & $59(10.9)$ & $<0.001$ & $<0.001$ \\
\hline Ever smoker & $335(83.1)$ & $110(79.1)$ & 0.290 & $332(61.3)$ & $<0.001$ & $<0.001$ \\
\hline Pack year $($ mean $\pm S D)$ & $18.8(18.3)$ & I4.I (16.7) & 0.008 & $8.6(12.5)$ & $<0.001$ & $<\mathbf{0 . 0 0 I}$ \\
\hline Productive cough & $205(52.4)$ & $55(40.1)$ & 0.013 & $139(25.6)$ & $<0.001$ & 0.002 \\
\hline $\mathrm{mMRC} \geq 2$ & $94(23.3)$ & $21(15.1)$ & $0.04 I$ & $34(6.3)$ & $<0.001$ & 0.001 \\
\hline Any respiratory symptoms & $302(74.9)$ & $98(70.5)$ & 0.305 & $225(41.6)$ & $<0.001$ & $<\mathbf{0 . 0 0 I}$ \\
\hline Allergic rhinitis & $90(22.4)$ & $37(26.6)$ & 0.317 & $135(25.0)$ & 0.372 & 0.687 \\
\hline Physician diagnosis of & 45 (1I.2) & $22(15.8)$ & 0.345 & $19(3.5)$ & $<0.001$ & $<0.001$ \\
\hline asthma & & & & & & \\
\hline Exacerbation, any & $84(20.8)$ & $24(17.3)$ & 0.363 & $36(6.6)$ & $<0.001$ & $<0.001$ \\
\hline Exacerbations, frequent & $34(8.4)$ & II (7.9) & 0.847 & $12(2.2)$ & $<0.001$ & $<\mathbf{0 . 0 0 I}$ \\
\hline Heart disease & $99(24.6)$ & $27(19.4)$ & 0.216 & $101(18.6)$ & 0.027 & 0.827 \\
\hline Diabetes mellitus & 45 (II.2) & $16(11.5)$ & 0.912 & $63(11.6)$ & 0.506 & 0.970 \\
\hline Anxiety/depression & $51(12.7)$ & $21(15.1)$ & 0.463 & $44(8.1)$ & 0.022 & 0.012 \\
\hline \multirow[t]{2}{*}{ Mortality } & $163(40.4)$ & $44(31.7)$ & 0.066 & $163(30.1)$ & 0.001 & 0.718 \\
\hline & $n=333$ & $n=118$ & & $n=45 I$ & & \\
\hline \multicolumn{7}{|l|}{ Women } \\
\hline Age (mean $\pm S D)$ & $65.4(11.3)$ & $65.2(11.4)$ & 0.910 & $65.0(11.3)$ & 0.644 & 0.838 \\
\hline $\mathrm{BMI}($ mean $\pm \mathrm{SD})$ & $25.5(4.3)$ & $26.6(4.9)$ & 0.017 & $26.6(4.3)$ & $<0.001$ & 0.916 \\
\hline $\mathrm{FEV}_{1} \%$ pred $($ mean $\pm \mathrm{SD})$ & $73.9(17.2)$ & $85.0(15.0)$ & $<0.001$ & $93.2(13.1)$ & $<0.001$ & 0.001 \\
\hline Nonsmoker & $98(29.4)$ & $43(36.4)$ & & $265(58.8)$ & & \\
\hline Ex-smoker & $102(30.6)$ & $50(42.4)$ & & $119(26.4)$ & & \\
\hline Current smoker & $133(39.9)$ & $25(21.2)$ & 0.001 & $67(14.9)$ & $<\mathbf{0 . 0 0 1}$ & $<0.001$ \\
\hline Ever smoker & $235(70.6)$ & $75(63.6)$ & 0.158 & $186(41.2)$ & $<0.001$ & $<\mathbf{0 . 0 0 1}$ \\
\hline Pack years $($ mean $\pm S D)$ & $13.9(14.0)$ & $8.8(11.5)$ & $<0.001$ & $4.9(9.0)$ & $<0.001$ & 0.001 \\
\hline Productive cough & I $33(40.7)$ & $32(27.1)$ & 0.014 & $94(21.4)$ & $<\mathbf{0 . 0 0 1}$ & 0.141 \\
\hline $\mathrm{mMRC} \geq 2$ & $115(34.5)$ & $33(28.0)$ & 0.192 & $60(13.3)$ & $<0.001$ & $<\mathbf{0 . 0 0 1}$ \\
\hline Any respiratory symptoms & $249(75.0)$ & $82(69.5)$ & 0.244 & $207(45.9)$ & $<0.001$ & $<0.00 \mathrm{I}$ \\
\hline Allergic rhinitis & $119(35.7)$ & $47(39.8)$ & 0.428 & $109(24.2)$ & $<0.001$ & 0.001 \\
\hline $\begin{array}{l}\text { Physician diagnosis of } \\
\text { asthma }\end{array}$ & 37 (II.I) & $14(11.9)$ & 0.850 & $18(4.0)$ & $<0.001$ & 0.004 \\
\hline Exacerbation, any & $76(22.8)$ & $22(18.6)$ & 0.344 & $53(11.8)$ & $<0.001$ & 0.049 \\
\hline Exacerbation, frequent & $32(9.6)$ & $8(6.8)$ & 0.353 & $18(4.0)$ & 0.001 & 0.197 \\
\hline Heart disease & $40(12.0)$ & $19(16.1)$ & 0.258 & $59(13.1)$ & 0.656 & 0.396 \\
\hline Diabetes mellitus & $21(6.3)$ & $9(7.6)$ & 0.621 & $35(7.8)$ & 0.434 & 0.961 \\
\hline Anxiety/depression & $85(25.5)$ & $20(16.9)$ & 0.058 & $90(20.0)$ & 0.064 & 0.462 \\
\hline Mortality & $105(31.5)$ & $29(24.6)$ & 0.155 & $99(22.0)$ & 0.003 & 0.543 \\
\hline
\end{tabular}

Notes: Data presented as $n$ (\%) unless stated otherwise. Significant $P$-values in bold. ${ }^{\text {FFEV }}$ /best of FVC and VC $<0.70$; ${ }^{b} F E V_{1} /$ best of $F V C$ and VC $\geq 0.70$; ${ }^{c}$ pre- not post-BD obstructive; ' ${ }^{\circ}$ comparing COPD and pre- not post-BD obstructive; 'comparing COPD and nonobstructive; 'comparing pre- not post-BD obstructive and nonobstructive. Abbreviations: BD, bronchodilator; BMI, body mass index; $\mathrm{FEV}_{1}$, forced expiratory volume in I s; FVC, forced vital capacity; mMRC, modified Medical Research Council; pred, predicted; SD, standard deviation; VC, vital capacity.

remained associated with an increased risk for death, 1.24 (1.01-1.53) and $2.37(1.69-3.30)$.

\section{Discussion}

In this population-based study, physician diagnosis of asthma before the age of 40 years reported allergic rhinitis, any respiratory symptoms, exacerbations, and comorbidities were equally common among subjects with COPD and pre- not post-BD obstruction. However, mortality was higher among subjects with COPD than pre- not post-BD obstruction, and those with COPD, specifically GOLD 2 and GOLD 3-4, but not pre- not post-BD obstruction, had an increased risk 
Table 3 Basic characteristics at recruitment, comparing men and women within the groups COPD, pre- not post-BD obstructive, and nonobstructive

\begin{tabular}{|c|c|c|c|c|c|c|c|c|c|}
\hline \multirow[t]{5}{*}{ Variables } & \multicolumn{6}{|c|}{ Airway obstruction ${ }^{a}$} & \multicolumn{3}{|c|}{ Nonobstructive $^{b}$} \\
\hline & \multirow{2}{*}{\multicolumn{2}{|c|}{ COPD }} & \multirow[t]{4}{*}{$P$-value ${ }^{d}$} & \multirow{2}{*}{\multicolumn{2}{|c|}{$\begin{array}{l}\text { Pre- not post-BD } \\
\text { obstructive }^{c}\end{array}$}} & \multirow[t]{4}{*}{$P$-value ${ }^{e}$} & & & \multirow[t]{4}{*}{$P$-value $f$} \\
\hline & & & & & & & & & \\
\hline & \multirow{2}{*}{$\begin{array}{l}\text { Men } \\
n=403\end{array}$} & \multirow{2}{*}{$\frac{\text { Women }}{n=333}$} & & \multirow{2}{*}{$\begin{array}{l}\text { Men } \\
n=139\end{array}$} & \multirow{2}{*}{$\begin{array}{l}\text { Women } \\
\mathrm{n}=118\end{array}$} & & \multirow{2}{*}{$\begin{array}{l}\text { Men } \\
n=542\end{array}$} & \multirow{2}{*}{$\begin{array}{l}\text { Women } \\
\mathrm{n}=45 \mathrm{I}\end{array}$} & \\
\hline & & & & & & & & & \\
\hline Age (mean \pm SD) & $65.5(10.7)$ & $65.4(11.3)$ & 0.889 & $61.6(12.8)$ & $65.2(11.4)$ & 0.017 & $64.1(11.3)$ & $65.0(11.3)$ & 0.215 \\
\hline BMI (mean \pm SD) & $25.9(3.5)$ & $25.5(4.3)$ & 0.138 & $27.3(4.4)$ & $26.6(4.9)$ & 0.228 & $26.6(3.4)$ & $26.6(4.3)$ & 0.946 \\
\hline $\mathrm{FEV}_{1} \%$ pred $($ mean $\pm \mathrm{SD})$ & $72.8(17.6)$ & $73.9(17.3)$ & 0.389 & $84.1(13.4)$ & $85.0(14.9)$ & 0.635 & $92.4(12.9)$ & $93.2(13.1)$ & 0.327 \\
\hline Nonsmoker & $68(16.9)$ & $98(29.4)$ & & 29 (20.9) & $43(36.4)$ & & $210(38.7)$ & $265(58.8)$ & \\
\hline Ex-smoker & $189(46.9)$ & $102(30.6)$ & & $83(59.7)$ & $50(42.4)$ & & $273(50.4)$ & $119(26.4)$ & \\
\hline Current smoker & $146(36.2)$ & 133 (39.9) & $<0.001$ & $27(19.4)$ & $25(21.2)$ & 0.009 & $59(10.9)$ & $67(14.9)$ & $<\mathbf{0 . 0 0 I}$ \\
\hline Ever smoker & $335(83.1)$ & $235(70.6)$ & $<0.001$ & I I0 (79.I) & $75(63.6)$ & 0.006 & $332(6 \mid .3)$ & I86 (4I.2) & $<\mathbf{0 . 0 0 I}$ \\
\hline Pack year $($ mean $\pm S D)$ & $18.8(18.3)$ & $13.9(14.0)$ & $<0.001$ & I4.I (16.7) & $8.8(11.5)$ & 0.003 & $8.6(I 2.5)$ & $4.9(9.0)$ & $<0.001$ \\
\hline Productive cough & $205(50.9)$ & I33 (39.9) & 0.003 & $55(39.6)$ & $32(27.1)$ & 0.036 & $139(25.6)$ & $94(20.8)$ & 0.075 \\
\hline $\mathrm{mMRC} \geq 2$ & $94(23.3)$ & 115 (34.5) & 0.001 & $21(15.1)$ & $33(28.0)$ & 0.012 & $34(6.3)$ & $60(13.3)$ & $<\mathbf{0 . 0 0 I}$ \\
\hline Any respiratory symptoms & $302(74.9)$ & $249(75.0)$ & 0.985 & $98(70.5)$ & $82(69.5)$ & 0.860 & $225(4 I .6)$ & $207(45.9)$ & 0.173 \\
\hline Allergic rhinitis & $90(22.4)$ & $119(35.7)$ & $<0.001$ & $37(26.6)$ & $47(39.8)$ & 0.024 & $135(25.0)$ & $109(24.2)$ & 0.775 \\
\hline Physician diagnosis of asthma & 45 (II.2) & 37 (II.I) & 0.955 & $22(15.8)$ & $14(11.9)$ & 0.588 & $19(3.5)$ & $18(4.0)$ & 0.836 \\
\hline Exacerbation, any & $84(20.8)$ & $76(22.8)$ & 0.517 & $24(17.3)$ & $22(18.6)$ & 0.774 & $36(6.6)$ & $53(11.8)$ & 0.005 \\
\hline Exacerbation, frequent & $34(8.4)$ & $32(9.6)$ & 0.579 & II (7.9) & $8(6.8)$ & 0.729 & $12(2.2)$ & $18(4.0)$ & 0.103 \\
\hline Heart disease & $99(24.6)$ & $40(12.0)$ & $<0.001$ & $27(19.4)$ & $19(16.1)$ & 0.489 & I0I (I8.6) & $59(13.1)$ & 0.018 \\
\hline Diabetes mellitus & $45($ (II.2) & $21(6.3)$ & 0.022 & $16(11.5)$ & $9(7.6)$ & 0.295 & $63(11.6)$ & $35(7.8)$ & 0.042 \\
\hline Anxiety/depression & $5 I(I 2.7)$ & $85(25.5)$ & $<0.001$ & $21(15.1)$ & $20(16.9)$ & 0.688 & $44(8.1)$ & $90(20.0)$ & $<0.001$ \\
\hline Mortality & $163(40.4)$ & $105(3 \mid .5)$ & 0.012 & $44(31.7)$ & $29(24.6)$ & 0.210 & $163(30.1)$ & $99(22.0)$ & 0.004 \\
\hline
\end{tabular}

Notes: Data presented as n (\%) unless stated otherwise. Significant $P$-values in bold. a FEV /best of FVC and VC $<0.70$; ${ }^{b} F E V$, best of FVC and VC $\geq 0.70$; ${ }^{\mathrm{C}}$ pre- not post-BD obstructive; 'comparing men and women with COPD; ${ }^{\mathrm{e}}$ comparing men and women with pre- not post-BD obstruction; 'comparing nonobstructive men and women.

Abbreviations: BD, bronchodilator; BMI, body mass index; FEV , forced expiratory volume in I s; FVC, forced vital capacity; mMRC, modified Medical Research Council; pred, predicted; SD, standard deviation; VC, vital capacity.

for death when compared with nonobstructive subjects. The increased risk for death for GOLD stage 2 and higher persisted when adjusted for not only common confounders but also comorbidities. The widespread uses of merely pre-BD spirometry in clinical practice will thus not only contribute to misclassification of subjects with reversible airway obstruction as COPD but also interfere with prognostic evaluation.

Every fourth subject with airway obstruction was no longer obstructive after bronchodilation and classified as
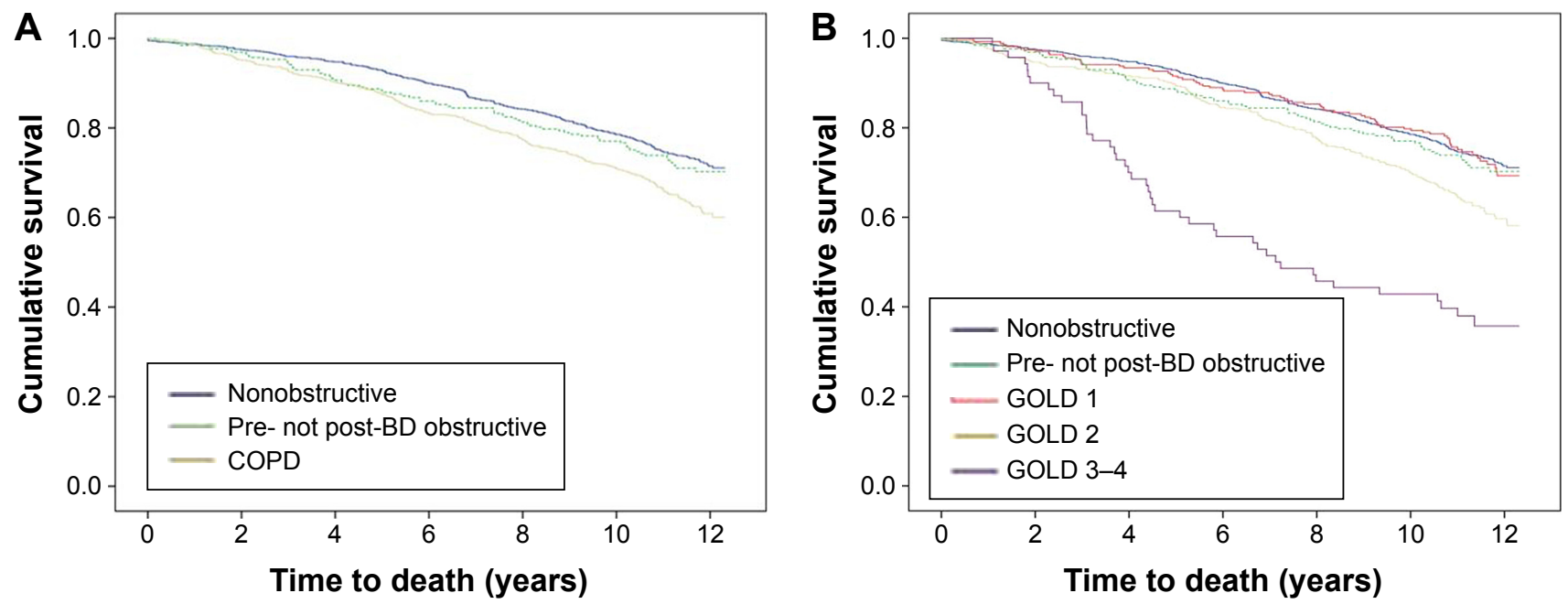

Figure 2 Survival among nonobstructive, pre-BD obstructive, and COPD (A) illustrated by Kaplan-Meier curves, (B) divided into GOLD I, GOLD 2, and GOLD 3-4 illustrated by Kaplan-Meier curves.

Abbreviations: BD, bronchodilator; GOLD, Global Initiative for Chronic Obstructive Lung Disease. 
Table 4 Unadjusted analyses of risk for death, expressed as HR $(95 \% \mathrm{Cl})$, among subjects with pre- not post-BD obstructive and COPD, also divided into GOLD stages, in comparison with nonobstructive, ${ }^{a}$ in the total study population, also stratified by age group and sex

\begin{tabular}{|c|c|c|c|c|c|}
\hline \multirow[t]{3}{*}{ Variable } & \multicolumn{5}{|l|}{ Airway obstructionc } \\
\hline & Pre- not post-BD obstructive ${ }^{b}$ & COPD $^{d}$ & GOLD Id & GOLD $2^{\mathrm{d}}$ & GOLD $3+4^{d}$ \\
\hline & HR (95\% Cl) & HR (95\% Cl) & HR (95\% Cl) & HR (95\% Cl) & HR (95\% CI) \\
\hline Total population & $1.10(0.85-1.42)$ & $1.48(1.24-1.75)$ & $1.02(0.79-1.32)$ & $1.55(1.26-1.89)$ & $3.59(2.6 \mathrm{I}-4.94)$ \\
\hline Age $\leq 60$ years & $2.12(1.02-4.40)$ & $1.21(0.63-2.32)$ & $0.49(.015-1.64)$ & $1.54(0.73-3.27)$ & 5.01 (1.49-16.79) \\
\hline Age $>60$ years & $1.00(0.76-1.32)$ & $1.44(1.20-1.7 I)$ & $1.16(0.86-1.46)$ & $1.43(1.16-1.76$ & $2.97(2.13-4.14)$ \\
\hline Men & $1.08(0.77-1.50)$ & $1.45(1.17-1.80)$ & $\mathrm{I} .04(0.75-\mathrm{I} .44)$ & $1.52(1.17-1.97)$ & $3.13(2.08-4.77)$ \\
\hline Women & $1.14(0.76-1.73)$ & $1.53(1.16-2.01)$ & $0.98(0.63-I .5 I)$ & $1.60(1.17-2.21)$ & $4.30(2.60-7.11)$ \\
\hline
\end{tabular}

Notes: Significant values in bold. aReference group: nonobstructive, $\mathrm{FEV}, \mathrm{VC} \geq 0.7$; ${ }^{\mathrm{b}}$ pre- but not post-BD airway obstruction; 'airway obstruction, $\mathrm{FEV}$ /best of FVC and $\mathrm{VC}$ $<0.7$; ${ }^{\circ}$ COPD analyzed as GOLD stages.

Abbreviations: BD, bronchodilator; $\mathrm{Cl}$, confidence interval; $\mathrm{FEV}$, forced expiratory volume in I s; GOLD, Global Initiative for Chronic Obstructive Lung Disease; HR, hazard ratio; VC, vital capacity.

pre- not post-BD obstructive, and this could not be explained by any significant difference in the presence of reported asthma or allergic rhinitis. The observed proportion with prenot post-BD obstruction is comparable to the $27 \%$ reported from a Norwegian population-based study ${ }^{25}$ but $<39 \%$ reported from the PLATINO study. ${ }^{26}$ According to current guidelines, post-BD spirometry is mandatory to confirm airway obstruction in COPD ${ }^{2}$ however, among subjects with recently diagnosed COPD, spirometry was found in only $32 \%-59 \%$ of the medical records and post-BD spirometry was far from always performed. ${ }^{7,9}$ There is an underutilization of spirometry in clinical practice, and when performed often merely pre- but not post-BD spirometry, which contributes to underdiagnosis as well as misclassification of COPD.

In the Lung Health Study, including a selected population of "healthy current smokers" with airway obstruction, both pre- and post-BD obstruction predicted mortality, ${ }^{27}$ while in this population-based study COPD but not pre- not post-BD obstruction was associated with increased mortality. It has clearly been established that subjects with COPD have increased mortality, ${ }^{28-30}$ and that disease severity, assessed as $\mathrm{FEV}_{1}$, is associated with increased mortality. ${ }^{28,31}$ Comorbidities, such as cardiovascular disease, diabetes, and anxiety/ depression, are common in COPD; ${ }^{32}$ at least $68 \%$ suffer from any of these comorbidities, ${ }^{16}$ and comorbidities may also contribute to increased mortality. ${ }^{14}$ Also in the present study, the risk for death increased by disease severity; GOLD grade 2 and higher had an increased risk for death not only independent of age, sex, and smoking habits, but moreover, independent also of comorbidities such as heart disease, diabetes, and anxiety/depression; GOLD 2 had 25\% increased risk for death, and in GOLD 3-4 the risk was more than doubled when compared to nonobstructive subjects. However, there was a discrepant pattern among subjects aged $\leq 60$ years, with an increased risk for death among those with pre- not post-BD airway obstruction, but not COPD, which has previously not been described. One explanation may be that the cause of death among people aged $>60$ and $<60$ years differs, and airway disease itself may not be the cause of death in pre- not post-BD obstructive but rather in severe COPD.

The clinical presentation of subjects with COPD and pre- not post-BD airway obstruction was rather similar with respect to reported respiratory symptoms, allergic rhinitis, and physician diagnosis of asthma even though productive cough as well as dyspnea ( $\mathrm{mMRC} \geq 2$ ) were more common among subjects with COPD. Misclassification due to the reported common use of merely pre-BD spirometry ${ }^{7,9}$ contributes to incorrect diagnostics of obstructive airway diseases. Clinical presentation is not sufficient to identify COPD in the population and the results highlight the need for the implementation of post-BD spirometry in clinical practice. A correct diagnosis is indeed important for the patient and also the basis for medication according to guidelines, ${ }^{2}$ and furthermore important when evaluating prognosis.

Sex is most often evaluated as a risk factor in obstructive airway disease, even though sex-stratified analyses are required to evaluate possible different risk factor patterns between sexes. ${ }^{33}$ It has been indicated that women are more vulnerable to tobacco smoke exposure and could develop more severe COPD than men. ${ }^{15}$ According to the Swedish National Board of Health and Welfare cause of death statistics, the number of deaths due to COPD has been gradually increasing among women during the last 15 years, actually now exceeding that of men, in which it has leveled off and been on the same level during the last 10 years. ${ }^{34}$ Even though men had higher mortality than women in the groups COPD and nonobstructive in this 
study, analyses stratified for sex revealed a similar pattern in both sexes, indicating an increased risk for death among both men and women with COPD independent of common confounders as well as comorbidities when compared with nonobstructive subjects.

This population-based study includes a large, wellcharacterized cohort of subjects with airway obstruction, with a distribution by GOLD stage among those fulfilling the spirometric criteria for COPD corresponding well to findings of other population-based studies. ${ }^{4}$ The statistical power was large enough to demonstrate an increased mortality already among subjects with moderate COPD, GOLD 2. Furthermore, the questionnaire is well validated and spirometry was performed according to guidelines. However, the study was designed around the shift of the millennium, just after the launch of the GOLD guidelines, recommending the use of a post-BD fixed ratio, $\mathrm{FEV}_{1} / \mathrm{FVC}<0.70$, to define airway obstruction in COPD and the fixed ratio may contribute to an overdiagnosis of COPD among the elderly. ${ }^{35}$ LLN is considered more physiological to define airway obstruction, and post-BD LLN is nowadays recommended to define COPD in epidemiological studies. ${ }^{3}$ By using LLN, the prevalence of COPD will be reduced compared to using the fixed ratio in population-based studies. ${ }^{36}$ In contrast, most current treatment guidelines are still based on the fixed ratio; why it remains important to evaluate also the fixed ratio definition of airway obstruction in population-based studies, even though the age dependence of the fixed ratio is important when interpreting the results. Another limitation of the current study is that asthma diagnosis as well as comorbidities was self-reported and not confirmed by medical records.

\section{Conclusion}

In this population-based study, clinical presentation could not clearly differentiate subjects with pre- not post-BD airway obstruction and COPD, but every fourth subject would be misclassified as obstructive if merely pre-BD spirometry was performed. Despite similar clinical characteristics and the burden of comorbidities among those with pre- not post-BD airway obstruction and COPD, only those with COPD had an increased risk for death, specifically in GOLD stage 2 and higher when compared to nonobstructive subjects, and the pattern was similar in both sexes. The results highlight the need for the implementation of post-BD spirometry in clinical practice to avoid misclassification of COPD; a correct diagnosis is not only the basis for treatment but also of prognostic importance.

\section{Acknowledgments}

The authors thank The Swedish Heart-Lung Foundation, Norrbotten County Council, and Visare Norr for financial support over the years. Thanks to Ann-Christin Jonsson and Sigrid Sundström for data collection. Thanks are also due to Helena Backman for consultation and constructing of databases, and the assistance of Ola Bernhoff for organizing the computerization of data is acknowledged. The present address of Sami Sawalha is Lung and Allergy Department, Sunderby Hospital, Luleå, Sweden.

\section{Disclosure}

The authors report no conflicts of interest in this work. The authors alone are responsible for the content and writing of the paper.

\section{References}

1. Fabbri LM, Rabe KF. From COPD to chronic systemic inflammatory syndrome? Lancet. 2007;370(9589):797-799.

2. The Global Initiative for Chronic Obstructive Lung Disease (GOLD). Global strategy for the diagnosis, management, and prevention of chronic obstructive pulmonary disease; 2016. Available from: http:// goldcopd.org/global-strategy-diagnosis-management-prevention-copd2016/. Accessed November 1, 2016.

3. Bakke PS, Rönmark E, Eagan T, et al. Recommendations for epidemiological studies on COPD. Eur Respir J. 2011;38(6):1261.

4. Lindberg A, Bjerg A, Ronmark E, Larsson LG, Lundback B. Prevalence and underdiagnosis of COPD by disease severity and the attributable fraction of smoking. Report from the Obstructive Lung Disease in Northern Sweden Studies. Respir Med. 2006;100(2):264-272.

5. Sobradillo V, Miravitlles M, Jimenez CA, et al. Epidemiological study of chronic obstructive pulmonary disease in Spain (IBERPOC): prevalence of chronic respiratory symptoms and airflow limitation. Arch Bronconeumol. 1999;35(4):159-166.

6. Pena VS, Miravitlles M, Gabriel R, et al. Geographic variations in prevalence and underdiagnosis of COPD: results of the IBERPOC multicentre epidemiological study. Chest. 2000;118(4):981-989.

7. Arne M, Lisspers K, Ställberg B, et al. How often is diagnosis of COPD confirmed with spirometry? Respir Med. 2010;104(4):550-556.

8. Han MK, Agusti A, Calverley PM, et al. Chronic obstructive pulmonary disease phenotypes: the future of COPD. Am J Respir Crit Care Med. 2010;182(5):598-604.

9. Han MK, Kim MG, Mardon R, et al. Spirometry utilization for COPD: how do we measure up? Chest. 2007;132(2):403-409.

10. Sterk PJ. Let's not forget: the GOLD criteria for COPD are based on

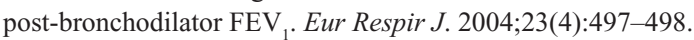

11. Richter DC, Joubert JR, Nell H, Schuurmans MM, Irusen EM. Diagnostic value of post-bronchodilator pulmonary function testing to distinguish between stable, moderate to severe COPD and asthma. Int J Chron Obstruct Pulmon Dis. 2008;3(4):693-699.

12. Atlantis E, Fahey P, Cochrane B, Smith S. Bidirectional associations between clinically relevant depression or anxiety and COPD: a systematic review and meta-analysis. Chest. 2013;144(3):766.

13. Laurin C, Moullec G, Bacon SL, Lavoie KL. Impact of anxiety and depression on chronic obstructive pulmonary disease exacerbation risk. Am J Respir Crit Care Med. 2012;185(9):918.

14. Sin DD, Anthonisen NR, Soriano JB, Agusti AG. Mortality in COPD: role of comorbidities. Eur Respir J. 2006;28(6):1245-1257. 
15. Sörheim I, Johannessen A, Gulsvik A, Bakke PS, Silverman EK, DeMeo DL. Gender differences in COPD: are women more susceptible to smoking effects than men? Thorax. 2010;65(6):480.

16. Anecchino C, Rossi E, Fanizza C, De Rosa M, Tognoni G, Romero M. Prevalence of chronic obstructive pulmonary disease and pattern of comorbidities in a general population. Int J Chron Obstruct Pulmon Dis. 2007;2(4):567-574.

17. Lindberg A, Lundback B. The Obstructive Lung Disease in Northern Sweden Chronic Obstructive Pulmonary Disease Study: design, the first year participation and mortality. Clin Respir J. 2008;2(Suppl 1): 64-71.

18. Lundback B, Nystrom L, Rosenhall L, Stjernberg N. Obstructive lung disease in northern Sweden: respiratory symptoms assessed in a postal survey. Eur Respir J. 1991;4(3):257-266.

19. Lundback B, Stjernberg N, Nystrom L, Lundback K, Lindstrom M, Rosenhall L. An interview study to estimate prevalence of asthma and chronic bronchitis. The obstructive lung disease in northern Sweden study. Eur J Epidemiol. 1993;9(2):123-133.

20. Lundback B, Stjernberg N, Nystrom L, et al. Epidemiology of respiratory symptoms, lung function and important determinants. Report from the Obstructive Lung Disease in Northern Sweden Project. Tuber Lung Dis. 1994;75(2):116-126.

21. Mahler DA, Wells CK. Evaluation of clinical methods for rating dyspnea. Chest. 1988;93(3):580.

22. Bestall JC, Paul EA, Garrod R, Garnham R, Jones PW, Wedzicha JA Usefulness of the Medical Research Council (MRC) dyspnoea scale as a measure of disability in patients with chronic obstructive pulmonary disease. Thorax. 1999;54(7):581.

23. American Thoracic Society. Standardization of spirometry, 1994 update American Thoracic Society. Am J Respir Crit Care Med. 1995;152(3): 1107-1136.

24. Backman H, Lindberg A, Oden A, et al. Reference values for spirometry - report from the Obstructive Lung Disease in Northern Sweden studies. Eur Clin Respir J. 2015;2:26375.

25. Johannessen A, Omenaas ER, Bakke PS, Gulsvik A. Implications of reversibility testing on prevalence and risk factors for chronic obstructive pulmonary disease: a community study. Thorax. 2005;60(10):842.
26. Montes de Oca M, Perez-Padilla R, Talamo C, et al. Acute bronchodilator responsiveness in subjects with and without airflow obstruction in five Latin American cities: the PLATINO study. Pulm Pharmacol Ther. 2010;23(1):29-35.

27. Mannino DM, Diaz-Guzman E, Buist S. Pre- and post-bronchodilator lung function as predictors of mortality in the Lung Health Study. Respir Res. 2011;12:136.

28. Lundback B, Eriksson B, Lindberg A, et al. A 20-year follow-up of a population study-based COPD cohort-report from the obstructive lung disease in Northern Sweden studies. COPD. 2009;6(4):263-271.

29. Stridsman C, Skar L, Hedman L, Ronmark E, Lindberg A. Fatigue affects health status and predicts mortality among subjects with COPD: report from the population-based OLIN COPD study. COPD. 2015;12(2):199-206.

30. Rycroft CE, Heyes A, Lanza L, Becker K. Epidemiology of chronic obstructive pulmonary disease: a literature review. Int J Chron Obstruct Pulmon Dis. 2012;7:457-494.

31. Mannino DM, Buist AS, Petty TL, Enright PL, Redd SC. Lung function and mortality in the United States: data from the First National Health and Nutrition Examination Survey follow up study. Thorax. 2003; 58(5):388-393.

32. Lindberg A, Larsson LG, Ronmark E, Lundback B. Co-morbidity in mild-to-moderate COPD: comparison to normal and restrictive lung function. COPD. 2011;8(6):421-428.

33. Becklake MR, Kauffmann F. Gender differences in airway behaviour over the human life span. Thorax. 1999;54(12):1119.

34. The Swedish National Board of Health and Welfare. Causes of Death 2010. Available from: http://www.socialstyrelsen.se/lists/artikelkatalog/ attachments/18394/2011-7-6.pdf. Accessed November 1, 2016.

35. Hardie JA, Buist AS, Vollmer WM, Ellingsen I, Bakke PS, Morkve O. Risk of over-diagnosis of COPD in asymptomatic elderly never-smokers. Eur Respir J. 2002;20(5):1117-1122.

36. Tilert T, Dillon C, Paulose-Ram R, Hnizdo E, Doney B. Estimating the US prevalence of chronic obstructive pulmonary disease using pre- and post-bronchodilator spirometry: the National Health and Nutrition Examination Survey (NHANES) 2007-2010. Respir Res. 2013; $14: 103$.
International Journal of COPD

\section{Publish your work in this journal}

The International Journal of COPD is an international, peer-reviewed journal of therapeutics and pharmacology focusing on concise rapid reporting of clinical studies and reviews in COPD. Special focus is given to the pathophysiological processes underlying the disease, intervention programs, patient focused education, and self management protocols.

\section{Dovepress}

This journal is indexed on PubMed Central, MedLine and CAS. The manuscript management system is completely online and includes a very quick and fair peer-review system, which is all easy to use. Visit http://www.dovepress.com/testimonials.php to read real quotes from published authors. 